\title{
Roundness Measurement in Spring Clamps Based on a Novel Particle Swarm Optimization
}

\author{
Xia Zhu and Renwen Chen
}

\begin{abstract}
One important inspection step in spring clamp is the determination of roundness value. Detect the circle from digital images is very important in computer vision. In view of clamp and the geometrical characteristics of the circular, a new optimization algorithm was proposed to extract image circle. Circle extraction is equivalent to the points search and extraction in the circle. Point search strategy is an optimization problem. Particle swarm optimization (PSO) is an effective global optimization method. Self-tuning parameters were achieved by using the improved particle swarm algorithm along with the geometric properties of the round, and detected effective point of band image so as to realize the recognition and detection of the circle. A large number of tests show that the proposed algorithm has good robustness and precision compared with the existing PSO algorithms. The main advantages of this method as compared with other methods include the considerable lower cost of facilities, easier maintenance and getting more accurate.
\end{abstract}

Index Terms-Spring clamp, diameter, circle detection, particle swarm optimization.

\section{INTRODUCTION}

Spring clamps is one of the important parts installed in the car. Spring clamps has been widely used and have a crucial effect on the performance of the car. So spring clamps' quality testing has always been a concern for manufacturers and users. For the same car, the diameter of the spring clamp must be kept constant. Diameter measurement, therefore, becomes the key problems in the process of spring clamp's detection. The diameter of spring clamp and the thickness of steel rod will be gotten through measuring the inner circle and outer circle.

With the rapid development of computer technology, the surface inspection based on machine vision is gradually becoming the mainstream of nondestructive testing. Its biggest advantage is not in contact with the products so the products to be measured do not produce any damage, which is very reliable.

Recently, several studies for industrial automation for measurement have been conducted by researchers. Such research revealed that machine vision inspection can be applied as an automatic instrument is practical conditions. Among the major important specifications for applying machine vision measurement are the followings: a) No need

Manuscript received July 22, 2014; revised December 29, 2014. This work was supported in part by the Priority Academic Program Development of Jiangsu Higher Education Institutions (PAPD).

The authors are with the State Key Laboratory of Mechanics and Control of Mechanical Structures, Nanjing University of Aeronautics \& Astronautics, Nanjing 210016, China (e-mail: snail2024@163.com, rwchen@nuaa.edu.cn). for precise measurement devices and their maintenance, b) reduction in the cost of skilled operators, c) elimination of the operator's measurement errors, d) and the high speed of the measurement techniques. These advantages have resulted into the substitution of machine vision measurement systems instead of common measuring methods in different industries.

In this study, a similar machine vision application is introduced as a new method for inspecting the spring clamps. From the image obtained by CCD, inner circle and outer circle of spring clamp are nearly round. As a result, diameter detection of spring clamp turns into circle detection. At present, circle detection methods include: loop integral differential method, a least squares fitting method, shape analysis method, circularity detection method, Hough transform, annealing algorithm, genetic algorithm and particle swarm optimization. ZOU [1] measured component size using the least square method, which reduces the noise influence on measurement accuracy to some extent, but for big noise, the result is not satisfactory. Hough transform, with its strong robustness, insensitivity to noise, is widely applied in the circle detection [2], [3]. But Hough transform has high computational complexity and occupy large amount of storage space, which can not satisfy the real-time request. To solve above problems, scholars put forward many improved methods [4]-[8], each have advantages and disadvantages. In this paper, circle detection was done by simple geometric principle: three points determinate a circle. When select the three points, the global search strategy was adapted. Compared with other evolutionary algorithms, PSO [9]-[13] has no special requirements for objective function, simple parameters setting, and small affect by target change, which is a simple and efficient parallel search. But traditional PSO can easily fall into premature convergence, which leads to lengthen computation time, increase calculation amount, lower efficiency. WEI [14] proposed IPSO on the basis of original PSO, which improves certain effect, but as for speed, convergence effect and accuracy it also can be improved.

This paper proposed a roundness measurement in spring clamps based on a novel PSO. Firstly, convert the clamp diameter detection into circle detection, and then search for the best solution the parameters of the circle in the search space, lastly get accurate diameter measurements. Simulation and practical experiments had verified the effectiveness of the method.

This paper is organized as follows. Section II describes the clamp diameter measurement systems. In Section III discussed the circle detection algorithm based on a novel particle swarm optimization algorithm. Section IV describes the roundness measurement using proposed PSO algorithm 
and shows the experimental setup and results. Our conclusions are presented in Section V.

\section{Clamp Diameter Measurement Systems}

The objective of this study is introducing a new and cost effective method for measuring roundness of clamp spring. By employing JAI CM030, the higher quality pictures can be obtained. This would decrease the perspective effect significantly. Furthermore, implementing a proper lighting system can simplify distinguishing between the pipe sections from the image background. Utilizing these methods helps to improve the measurement accuracy.

For irregular circular clamp with smooth surface, image quality will be affected by the light. In order to highlight the outline of clamp, this paper uses backlight way and uses a short white frosted glass as its background, while quadrilateral light source is placed directly parallel to the glass.

Dimension measurement system based on machine vision is mainly composed of camera calibration, image preprocessing, and diameter detection and so on. Firstly, the system catch real-time image and get clamp image (see Fig. 1). Secondly, image program is used to analysis image and give the test results at last. Image preprocessing mainly includes the grayscale image transformation, image denoising, image binarization and image edge detection and so on.

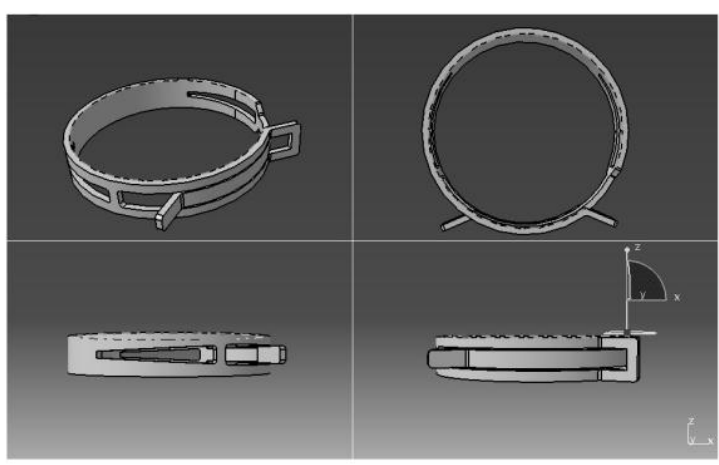

Fig. 1. Clamp schematic diagram.

First we extract image edge from binarization gray image. In the edges array $\Pi$ of the image, edge point is stored as an index to their position. $\left(x_{0}, y_{0}\right)$ is the coordinates of the center of the circle and $R$ is its radius. The general equation for circular is as follows:

$$
\begin{gathered}
\left(x_{i}-x_{0}\right)^{2}+\left(y_{i}-y_{0}\right)^{2}=R^{2} \\
\Pi=\left\{\left(x_{i}, y_{i}\right) \mid i=1,2, \ldots N, f\left(x_{i}, y_{i}\right)=1\right\}
\end{gathered}
$$

We can calculate the three parameters of the circle through the three edge points $\left(\left(x_{1}, y_{1}\right),\left(x_{2}, y_{2}\right),\left(x_{3}, y_{3}\right)\right)$.

$$
x_{0}=\frac{\left|\begin{array}{ll}
\left(x_{2}^{2}+y_{2}^{2}\right)-\left(x_{1}^{2}+y_{1}^{2}\right) & 2\left(y_{2}-y_{1}\right) \\
\left(x_{3}^{2}+y_{3}^{2}\right)-\left(x_{1}^{2}+y_{1}^{2}\right) & 2\left(y_{3}-y_{1}\right)
\end{array}\right|}{4\left[\left(x_{2}-x_{1}\right)\left(y_{3}-y_{1}\right)-\left(x_{3}-x_{1}\right)\left(y_{2}-y_{1}\right)\right]}
$$

$$
\begin{gathered}
y_{0}=\frac{\left|\begin{array}{ll}
\left(x_{2}^{2}+y_{2}^{2}\right)-\left(x_{1}^{2}+y_{1}^{2}\right) & 2\left(x_{2}-x_{1}\right) \\
\left(x_{3}^{2}+y_{3}^{2}\right)-\left(x_{1}^{2}+y_{1}^{2}\right) & 2\left(x_{3}-x_{1}\right)
\end{array}\right|}{4\left[\left(x_{3}-x_{1}\right)\left(y_{2}-y_{1}\right)-\left(x_{2}-x_{1}\right)\left(y_{3}-y_{1}\right)\right]} \\
R=\sqrt{\left(x_{i}-x_{0}\right)^{2}+\left(y_{i}-y_{0}\right)^{2}}, \quad i=1,2,
\end{gathered}
$$

At present, circle detection method basically includes: least squares, Hough transform, intelligent algorithm. The least-square method is high speed, but easily affected by edge noise, and easy to lead to high curvature deviation. Hough transform has strong robustness and is not sensitive to the noise characteristics, but it also has high computer amount and occupies large amount of storage space, which can not satisfy the real-time requirement. This paper proposes a novel particle swarm optimization algorithm to detect a circle.

\section{Circle Detection Algorithm BASED ON A Novel PARTICLE SWARM OPTIMIZATION ALGORITHM}

\section{A. A Novel Particle Swarm Optimization}

Dr. Kennedy first proposes particle swarm optimization algorithm [10]. He is inspired by birds' foraging behavior and does research on the behavior of birds and fish populations and so on.

Individual particle in group evaluate target position information by searching a multidimensional space. In the whole process, Particles share their optimal position and adjust their own speed and position using their own memory. Through share information and individual experience, particles correct their behavior to make the overall best, eventually find optimal solutions and local optimal solution. Thus in the original version particle moves by the following equation:

$$
\begin{gathered}
v_{i}(t+1)=\omega * v_{i}(t)+c_{1} * \operatorname{rand}() *\left(p_{i} \text { best }-x_{i}(t)\right) \\
+c_{2} * \operatorname{rand}() *\left(\text { gbest }-x_{i}(t)\right) \\
x_{i}(t+1)=x_{i}(t)+v_{i}(t+1)
\end{gathered}
$$

where $c_{1}$ and $c_{2}$ are two positive constants and $\operatorname{rand}()$ is a uniformly distributed random number in $[0,1] . \omega$ is an inertia weight in $[0.1,0.9]$.

Equation (6) consists of the following three parts: (1) the previously speeds of the particle $v_{i}(t) ;(2)$ the individual cognition, $c_{1} * \operatorname{rand}() *\left(p_{i}\right.$ best $\left.-x_{i}(t)\right)$ stands for a particle' self-thinking ability. (3) Social cognition, $c_{2} * \operatorname{rand}() *\left(\right.$ gbest $\left.-x_{i}(t)\right)$ reflect the information share ability between particles.

Under the effect of the above three parts, the particles constantly update their own position from the historical experience and information sharing in order to find the optimal solution.

In algorithm implementation, the particles have two actions: exploration and exploitation. Exploration refers that particles leave the original optimization procedure, turn to 
the new directions for global search; exploitation is refers to the particles continue optimizing in the local search. Inertia weight maintains the balance of local and global search ability. Shi [10], [11] and others do some research on inertia weight and they find that dynamic inertia weight can gain better optimization results than constant value. Now, a linear weighting strategy proposed by Shi was used in more paper, but it would result in time loss.

According to the above problems, we put forward an improved dynamic inertia weight particle swarm optimization (DIWPSO). Inertia weight adjustment formula is as follows:

$$
\begin{gathered}
\omega_{i}(t)=\frac{1}{1+\mathrm{e}^{\rho}} \\
\rho=\left|\frac{F_{m}^{i i}-F_{a v g}^{i i}}{F_{m}^{i i-1}-F_{a v g}^{i i-1}}\right|
\end{gathered}
$$

where $F_{a v g}^{i i}$ is average fitness value at time step $i i ; F_{m}^{i i}$ is optimal fitness value at time step $i i ; F_{m}^{i i-1}$ is average fitness value at time step $(i i-1) ; F_{a v g}^{i i-1}$ is optimal fitness value at time step $(i i-1)$

To test and verify the effect of the improved algorithm, we use famous benchmark problem [15] who were used in a large number of literatures for testing algorithm performance. The following equations give the definition, scope, and global optimal solution of functions.

1) BR-Branin function

$$
\begin{aligned}
& f_{B R}=\left(x_{2}-\frac{5}{4 \pi^{2}} x_{1}^{2}+\frac{5}{\pi} x_{1}-6\right)^{2} \\
& +10\left(1-\frac{1}{8 \pi}\right) \cos x_{1}+10 \\
& -5 \leq x_{1} \leq 10,0 \leq x_{2} \leq 15
\end{aligned}
$$

This function has three optimal coordinate $(9.425,2.425)$, $(3.142,2.275),(-3.142,12.275)$ and global minimum is 0.398 .

2) GP-Goldstein-Price function

$$
\begin{aligned}
& f_{G P}=\left[1+\left(x_{1}+x_{2}+1\right)^{2} \times\right. \\
& \left.\left(19-14 x_{1}+3 x_{1}^{2}-14 x_{2}+6 x_{1} x_{2}+3 x_{2}^{2}\right)\right] \times \\
& {\left[30+\left(2 x_{1}-3 x_{2}\right)^{2} \times\right.} \\
& \left.\left(18-32 x_{1}+12 x_{1}^{2}+48 x_{2}-36 x_{1} x_{2}+27 x_{2}^{2}\right)\right] \\
& -2 \leq x_{\mathrm{i}} \leq 2, i=1,2
\end{aligned}
$$

This function has one optimal coordinate $(0,-1)$, four local minimum points and global minimum is 3 .

3) RA-Rastrigin function

$$
\begin{gathered}
f_{R A}=x_{1}^{2}+x_{2}^{2}-\cos 18 x_{1}-\cos 18 x_{2}, \\
-1 \leq x_{i} \leq 1, i=1,2
\end{gathered}
$$

This function has one optimal coordinate $(0,0)$, fifty local minimum points and global minimum is -2 .
According to the above-mentioned three optimization problems, firstly, some comparisons were doing between DIWPSO and IPSO, PSO. Total group size is 20. For each test function, 100 runs were executed. Average minimum function value and standard deviation were listed in Table I. According to Table I, DIWPSO is relatively closer to theoretical optimal value than PSO and IPSO algorithm.

TABLE I: THE SiMULATION RESUlTS OF DIFFERENT ALGORITHMS UNDER

\begin{tabular}{cccc}
\multicolumn{4}{c}{ THE SAME ITERATIONS } \\
\hline function & PSO & IPSO & DIWPSO \\
\hline \multirow{2}{*}{$f_{B R}$} & 0.4102 & 0.429 & 0.3979 \\
& \pm 0.0135 & \pm 0.307 & \pm 0.005 \\
\hline \multirow{2}{*}{$f_{G P}$} & 3.2482 & 4.5727 & 3.0000 \\
& \pm 0.8959 & \pm 1.2156 & \pm 0.0214 \\
\hline \multirow{2}{*}{$f_{R A}$} & -2.003 & -1.9727 & -1.9967 \\
& \pm 0.0287 & \pm 0.0127 & \pm 0.03 \\
\hline
\end{tabular}

\section{B. Circle Detection Algorithm Based on Improved} Particle Swarm Optimization Algorithm

According to round geometric properties and Equation (1), we find that three points can determine a circle. Then how to choose the three points has always been the hot spot. Chen [4] proposed a hypothesis - test structure for choosing three points, while the deficiency was that parameters were too much in the program; Yu Dan [6] proposed randomized circle detection algorithm on the basis of Chen, it determined the search scope according to the connectivity, and large scope leaded to large computer time.

In this paper, we use another geometric feature of circle to select three points. Property is as follows: first we arbitrarily choose a point $A$ form circle; Line $A O$ will intersect the circle at point $B$. Then we choose another point $C$ on the left side of the circular $A O$. The circle is tangent to the line $l a$ and $l c$. The angle between lines $l c$ and line $A C$ is equal to the angle between lines $A C$ to $l a$. Then we choose another point $D$ on the right side of the circular $A O$. The circle is tangent to the line $l a$ and $l d$. The angle between lines $l d$ and line $A D$ is equal to the angle between lines $A D$ to la.

That is $\angle A C E=\angle C A F$ and $\angle A D N=\angle D A M$.

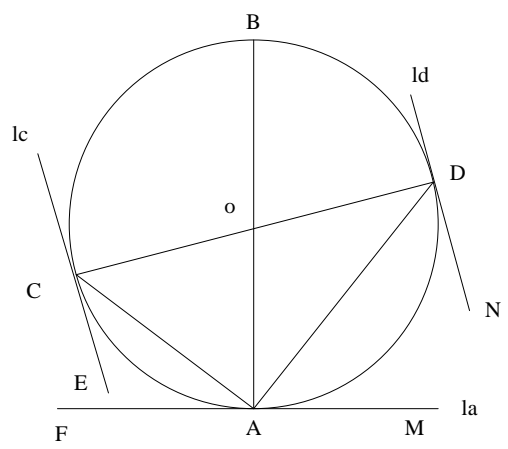

Fig. 2. Circle schematic diagram.

The algorithm implementation steps are as follows:

Step 1. Randomly select a point $A$ in the image; assume point $A$ is on the circle, the circle is tangent to the line $l a$ at point $A$.

Step 2. As shown in Fig. 2, start from point $A$, search within the scope of the right hemisphere. Once find point $D$, and then change the research direction. Again start from 
point $A$, search within the scope of the left hemisphere. Once find point $C$. So far, the three points is the random sampling points to determine circle.

Step 3. If another two points can not meet the requirement, then abandon point $A$, randomly select a point in the image, and restart from Step 1.

Point search strategy can be summed up in optimization problem, which is how to get best solution between accuracy and speed. Its essence is to optimize the similarity measure function, and determine the parameters for the space transformation. In this paper, we optimize point search strategy, expedite calculation speed and reduce the computation by using the proposed method. The algorithm implementation steps are shown in Fig. 3.

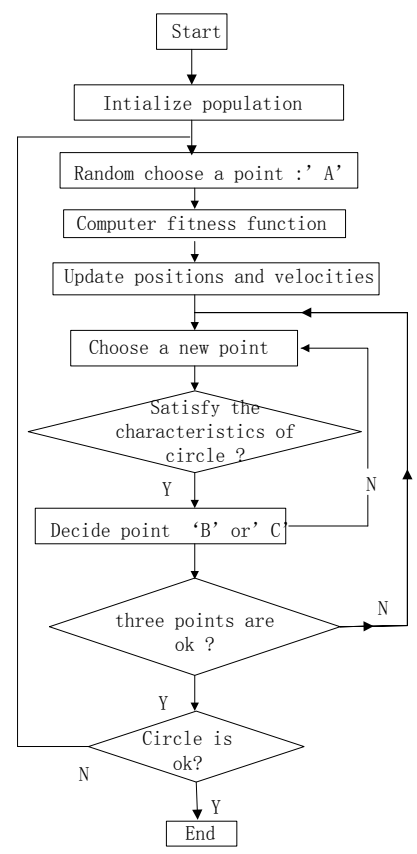

Fig. 3. The flow chart of circle detection algorithm based on improved particle swarm optimization.

\section{THE EXPERIMENTAL RESULTS AND ANALYSIS}

Several industrial parts are taken as test examples for demonstrating the proposed algorithm in Section III. Test platform was implemented in $\mathrm{C}++$ language, on a $\mathrm{PC}$ using a XP processor running at $2.2 \mathrm{GHz}$.

To verify DIWPSO performance in circle detection, we design some comparison experiments. Number of particles is 100, each algorithm runs 1500 times. The profiles of the measurement data sets for roundness assessments are given in Fig. 4-Fig. 8. In order to further compare the performance of three algorithms, the fitness function value and the time are listed in Table II.

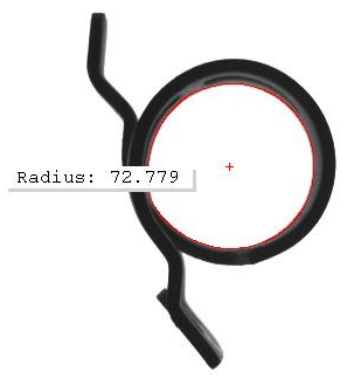

(a) The results based on PSO

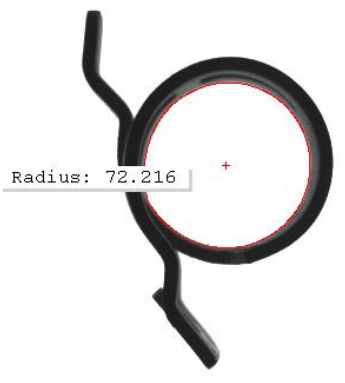

(b) The results based on IPSO

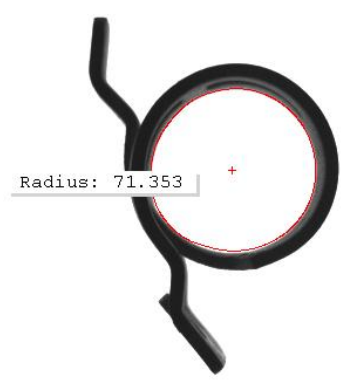

(c) The results based on DIWPSO

Fig. 4. Clamp detection (the image was captured from the actual test environment)

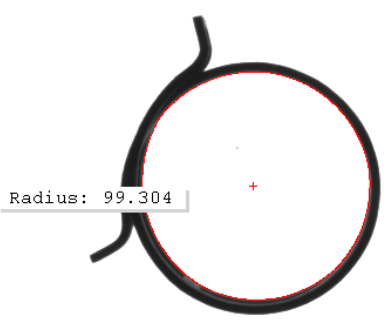

(a) The results based on PSO

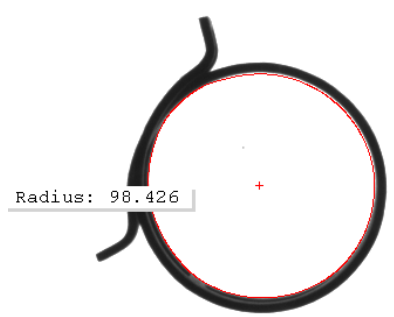

(b) The results based on IPSO

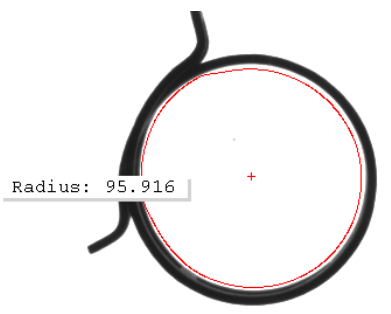

(c) The results based on DIWPSO

Fig. 5. Clamp detection (the image was captured from the actual test environment).

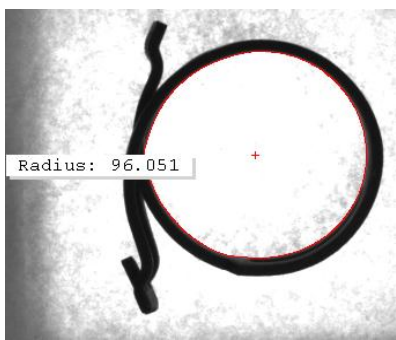

(a) The results based on PSO

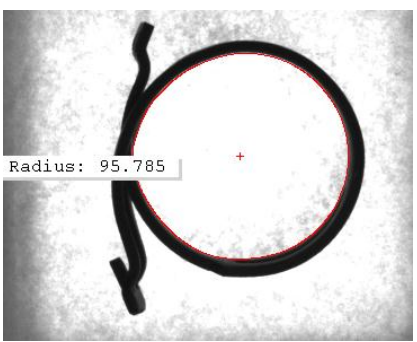

(b) The results based on IPSO

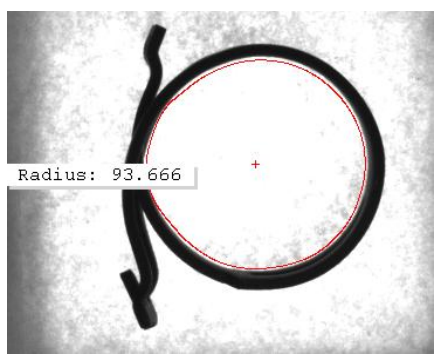

(c) The results based on DIWPSO

Fig. 6. Clamp detection (the image was captured from the actual test environment with large noise)

According to Fig. 4-Fig. 7, all test results of show that DIWPSO algorithm is satisfactory. Especially we can find that it can successfully detect the circle when dealing with a loud noise in Fig. 6 and Fig. 7. From Table II we can be concluded that the fitness function value of DIWPSO algorithm is less than PSO, IPSO. It also illustrate DIWPSO can obtain better testing results with less segmentation time. As for detection accuracy, DIWPSO test result is closer to 
actual value through compared with manual inspection late.

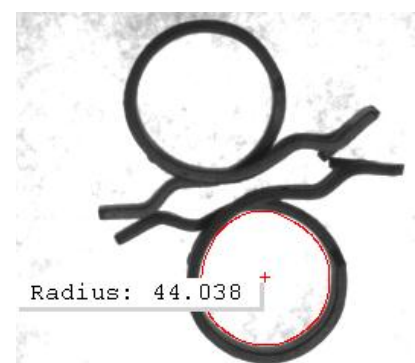

(a) The results based on PSO

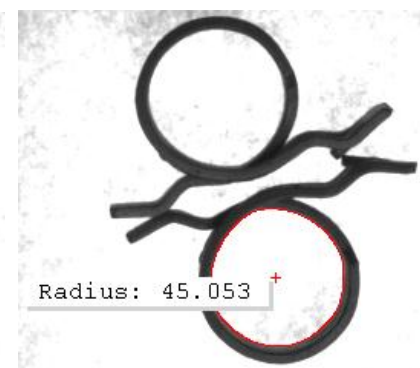

(b) The results based on IPSO

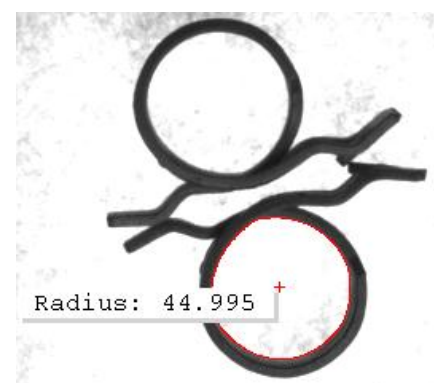

(c) The results based on DIWPSO

Fig. 7. Clamp detection (the image was captured from the actual test environment with little noise).

TABLE II: ThE OBJECTIVE Function VALUE AND THE RunNing TIME OF THE IMAGES

\begin{tabular}{cccc}
\hline Image & PSO & IPSO & DIWPSO \\
\hline 4 & $3.4565 \mathrm{E}+007$ & $5.4856 \mathrm{E}+006$ & $3.0148 \mathrm{E}+006$ \\
& 0.285 & 0.225 & 0.162 \\
\hline 5 & $4.8562 \mathrm{E}+007$ & $7.483 \mathrm{E}+006$ & $3.829 \mathrm{E}+006$ \\
& 0.491 & 0.294 & 0.207 \\
\hline 6 & $3.485 \mathrm{E}+008$ & $3.107 \mathrm{E}+008$ & $2.415 \mathrm{E}+008$ \\
& 2.149 & 2.001 & 1.427 \\
\hline 7 & $5.29 \mathrm{E}+008$ & $4.85 \mathrm{E}+008$ & $2.26 \mathrm{E}+008$ \\
& 1.852 & 1.024 & 0.428
\end{tabular}

(The first line is the objective function value; the second line is the running time (second))

To further validate the feasibility of the proposed algorithm, we detect hand-drawn circle. This can be shown in Fig. 8. We can say that the proposed algorithm can distinguish the hand-drawn circle form the other shapes present in the image.

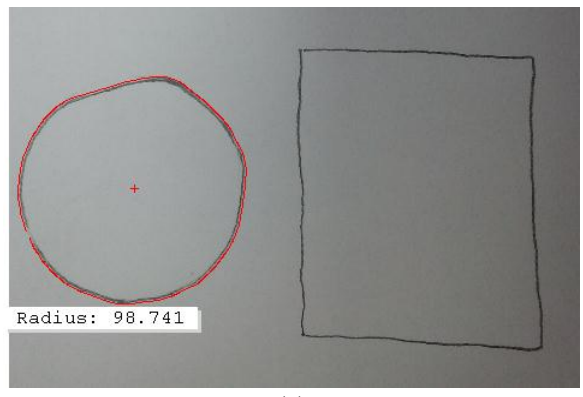

(a)

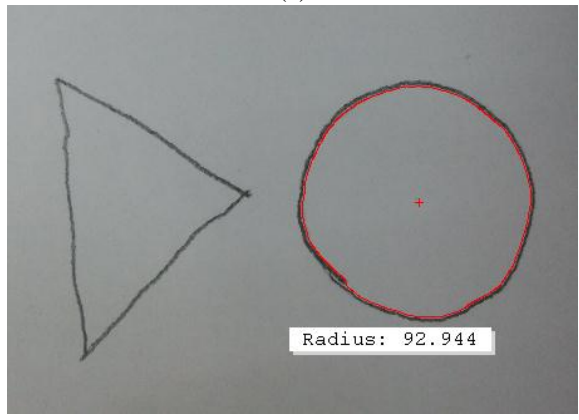

(b)

Fig. 8. Images of hand-drawn circle detection.
The above figures and analysis shows that circle diameter detection algorithm using DIWPSO algorithm can get a better results compared with previous PSO, IPSO algorithm. It has quick convergence rate, high detection quality and can reduce control parameters etc.

The proposed method can provide an effective measuring process compared to the former. In this paper, the total CPU time for image acquiring, threshold and boundary following is less than 1s. It has been suggested form the above results that the proposed method is a practical method for roundness measurement in industry.

\section{CONCLUSIONS}

Machine vision has been developed as a facility to mechanize measurement automatically. To improve speed and precision of the clamp diameter detection, firstly we converts the clamp diameter detection to the circle detection, then puts forward a circle detection algorithm based on a new particle swarm optimization algorithm, and finally verify the algorithm through the simulation experiment. At the same time, we do some comparisons with PSO, IPSO and so on. The experimental results show that the algorithm effectively enhances the precision and speed of circle detection, effectively improved the accuracy and speed of the clamp diameter detection which has great significance in the industry detection field. Furthermore, the proposed methodology can be easily applied to other roundness assessment instruments as well.

\section{ACKNOWLEDGMENT}

The authors thank the reviewers and editors for their corresponding contributions in making the paper more presentable. This research is supported by the Priority Academic Program Development of Jiangsu Higher Education Institutions (PAPD).

\section{REFERENCE}

[1] Y. M. Zou and B. Wang, "Fragmental ellipse fitting based on least square algorithm," Chinese Journal of Scientific Instrument, vol. 27, no. 7, pp. 808-812, 2006.

[2] F. Zhou, C. Yang, C. Wang et al., "Circle detection and its number identification in complex condition based on random Hough transform," Chinese Journal of Scientific Instrument, vol. 34, no. 3, pp. 622-628, 2013.

[3] Y. L. Huang, Y. T. Ye, Z. L. Chen et al., "New method of fast Hough transform for circle detection," Journal of Electronic Measurement and Instrument, vol. 24, no. 9, pp. 837-841, 2010.

[4] A. J. Chen, J. Z. Li, and D. D. Li, "Improved randomized algorithm for circle detection," Opto-Electronic Engineering, vol. 33, no. 12, pp. 91-95, 2006.

[5] L. Zuo, M. Li, X. W. Zhang et al., "Small-target detection in sea clutter based on improved Hough transform," Journal of Electronics G. Information Technology, vol. 34, no. 4, pp. 923-928, 2012.

[6] D. Yu, and W. Wei, "Improved randomized circle detection algorithm," Journal of Image and Graphics A, vol. 14, no. 8, pp. 1590-1593, 2009.

[7] M. K. Li and J. Guan, "Circle detection algorithm based on conformal geometric algebra and radon transform," Opto-Electronic Engineering, vol. 37, no. 4, pp. 72-76, 2010.

[8] L. Y. Jiang, "Fast randomized algorithm for detecting circles," Opto-Electronic Engineering, vol. 37, no. 1, pp. 71-75, 2010.

[9] R. C. Eberhart and J. Kennedy, "A new optimizer using particle swarm theory," in Proc. the Sixth International Symposium on Micromachine and Human Science, Nagoya, Japan, 1995, pp. 39-43.

[10] R. C. Eberhart and Y. Shi, "Particle swarm optimization: Developments, applications and resources," in Proc. the IEEE 
Congress on Evolutionary Computation, IEEE Press, Seoul, Korea, 2001.

[11] R. C. Eberhart and Y. Shi, "Comparing inertia weights and constriction factors in particle swarm optimization," in Proc. the IEEE Congress on Evolutionary Computation, San Diego, USA, 2000, pp. 84-88.

[12] E. Ozcan and C. K. Mohan, "Analysis of a simple particle swarm optimization system," Intelligent Engineering Systems through Artificial Neural Networks, pp. 253-258, 1998.

[13] E. Ozcan, and C. K. Mohan, "Particle swarm optimization: Surfing the waves," in Proc. the IEEE Congress on Evolutionary Computation, Washington, DC, USA, 1999.

[14] B. Z. Wei, Z. M. Zhao, and Y. Z. Song, "A novel algorithm IPSO algorithm and hybrid information," Journal of Optoelectronics, Laser, 2009, vol. 20, no. 9, no. 1271-1274.

[15] E. R. Davies, Computer and Machine Vision: Theory, Algorithm, Practicalities, Salt Lake City: Academic Press, 2012.

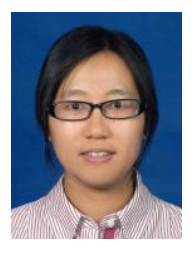

Xia Zhu was born in Jiangsu, China, in 1980. She received her undergraduate education in China University of Mining and Technology, China, in 2004. She received the master degree in Jiangnan University, China, in 2009. She is now in pursuit for Ph.D degree in measurement and testing technology \& instruments at State Key Laboratory of Mechanics and Control of Mechanical Structures, NUAA, Nanjing, China. Her current field of interest focuses on image processing, computer vision and intelligent monitoring and control.

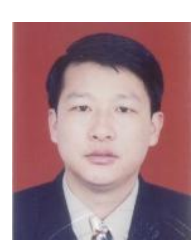

Renwen Chen was born in Hunan, China, in $1966 . \mathrm{He}$ graduated from Nanjing University of Aeronautics \& Astronautics (NUAA), Nanjing, China in 1991 and received his Ph.D degree from NUAA in 1999, both in measurement and testing technology \& instruments. He also spent half a year as a visiting scholar in University of California, Berkeley, USA. His current research interests are in the field of energy harvesting, active vibration control, wireless sensors network and intelligent monitoring and control. 